\title{
Anti-Ramsey Numbers in Complete k-Partite Graphs
}

\author{
Jili Ding, ${ }^{1}$ Hong Bian $(D), 1$ and Haizheng $\mathbf{Y u}^{2}$ \\ ${ }^{1}$ School of Mathematical Sciences, Xinjiang Normal University, Urumqi, Xinjiang 830054, China \\ ${ }^{2}$ College of Mathematics and System Sciences, Xinjiang University, Urumqi 830046, China
}

Correspondence should be addressed to Hong Bian; bh1218@163.com

Received 22 March 2020; Revised 29 July 2020; Accepted 17 August 2020; Published 7 September 2020

Academic Editor: Haipeng Peng

Copyright $\odot 2020$ Jili Ding et al. This is an open access article distributed under the Creative Commons Attribution License, which permits unrestricted use, distribution, and reproduction in any medium, provided the original work is properly cited.

The anti-Ramsey number $A R(G, H)$ is the maximum number of colors in an edge-coloring of $G$ such that $G$ contains no rainbow subgraphs isomorphic to $H$. In this paper, we discuss the anti-Ramsey numbers $A R\left(K_{p_{1}, p_{2}, \ldots, p_{k}}, \mathscr{T}_{n}\right), A R\left(K_{p_{1}, p_{2}, \ldots, p_{k}}, \mathscr{M}\right)$, and $A R\left(K_{p_{1}, p_{2}, \ldots, p_{k}}, \mathscr{C}\right)$ of $K_{p_{1}, p_{2}, \ldots, p_{k}}$, where $\mathscr{T}_{n}, \mathscr{M}$, and $\mathscr{C}$ denote the family of all spanning trees, the family of all perfect matchings, and the family of all Hamilton cycles in $K_{p_{1}, p_{2}, \ldots, p_{k}}$, respectively.

\section{Introduction}

Let $G$ be a graph, a $k$-edge-coloring of a graph $G=(V, E)$ is a mapping $c: E \longrightarrow C$, where $C$ is a set of colors, namely, $C=\{1,2, \ldots, k\}[1]$. A subgraph $H$ of an edge-colored graph $G$ is rainbow if all of its edges have different colors. An edgecolored graph is called rainbow graph if all the colors on the edges are distinct. A representing subgraph in an edgecoloring of $G$ is a spanning subgraph obtained by taking one edge of each color. The anti-Ramsey number $A R(G, H)$ is the maximum number of colors in an edge-coloring of $G$ with no rainbow copy of $H$. Rainbow coloring of graphs also has its application in practice. It comes from the secure communication of information between agencies of government. The anti-Ramsey number was introduced by Erdös, Simonovits, and Sos in 1973 [2]. It has been shown that the anti-Ramsey number $A R(G, H)$ is closely related to Turan number. The Turan number $\operatorname{ex}(n, H)$ is the maximum number of edges in a graph $G$ on $n$ vertices which does not contain any subgraph isomorphic to $H$. Erdös et al. conjectured that $A R\left(K_{n}, C_{k}\right)=n((k-2 / 2)+(1 / k-1))+$ $O(1)$, for every fixed $k \geq 3$ [2]. The conjecture is proved completely for all $k \geq 3$ in [3] by Montellano-Ballesteros and Neumann-Lara. The anti-Ramsey numbers for some other special graph classes in complete graphs have also been studied, including independent cycles [4], stars [5], spanning trees [6], and matchings [7, 8]. The anti-Ramsey problems for rainbow matchings, cycles, and trees in complete bipartite graphs have been studied in [9-11]. Some other graphs were also considered as the host graphs in anti-Ramsey problems, such as hypergraphs [12], hypecubes [13], plane triangulations [14], and planar graphs [15].

It is natural to consider that the anti-Ramsey problems for rainbow matchings, cycles, and trees in complete $k$-partite graphs. In this paper, we are interested in the antiRamsey numbers for spanning trees, perfect matchings, and Hamilton cycles in complete $k$-partite graphs. A complete $k$-partite graph is a graph whose vertices can be partitioned into $k$ different independent sets, and any two vertices from different independent sets are connected by an edge. A complete $k$-partite graph, with partitions $S_{1}, S_{2}, \ldots, S_{k}$, $k \geq 2$, is denoted by $K_{p_{1}, p_{2}, \ldots, p_{k}}$, without loss of generality; in the following, we always assume that $\left|S_{1}\right|=p_{1},\left|S_{2}\right|=p_{2}$, $\ldots,\left|S_{k}\right|=p_{k}, \quad p_{1} \geq p_{2} \geq \ldots \geq p_{k} \geq 2, \quad p_{1}+p_{2}+\ldots+p_{k}=$ $\sum_{i=1}^{n} p_{i}=n$. If $p_{1}=p_{2}=\ldots=p_{k}=1, G=K_{k}$ is a complete graph. Bialostocki and Voxman proved that $A R\left(K_{n}, T_{n}\right)=\left(\begin{array}{c}n-2 \\ 2\end{array}\right)+1$, where $T_{n}$ denotes the family of all spanning trees in $K_{n}$ [6]. The maximum number of colors in an edge-coloring of $K_{n}$ with no rainbow perfect matching (for even $n$ ) is $\left(\begin{array}{c}n-3 \\ 2\end{array}\right)+2$, when $n \geq 14$ [8]. 


\section{Main Result}

The family of all spanning trees in $K_{p_{1}, p_{2}, \ldots, p_{k}}$ is denoted by $\mathscr{T}_{n}$. The maximum number of colors in an edge-coloring of $K_{p_{1}, p_{2}, \ldots, p_{k}}$ not containing any rainbow spanning tree is denoted by $A R\left(K_{p_{1}, p_{2}, \ldots, p_{k}}, \mathscr{T}_{n}\right)$.

Theorem 1. If $\left|S_{1}\right|=p_{1},\left|S_{2}\right|=p_{2}, \ldots,\left|S_{k}\right|=p_{k}, p_{1} \geq p_{2} \geq \ldots$ $\geq p_{k} \geq 2, k \geq 2$, then

$$
A R\left(K_{p_{1}, p_{2}, \ldots, p_{k}}, \mathscr{T}_{n}\right) \geq \begin{cases}\sum_{1 \leq i<j \leq k} p_{i} p_{j}-2 \sum_{i=2}^{k} p_{i}+1, & p_{1}>p_{2}, \\ \sum_{1 \leq i<j \leq k} p_{i} p_{j}-2 \sum_{i=2}^{k} p_{i}+2, & p_{1}=p_{2} .\end{cases}
$$

Proof. Let $K_{p_{1}, p_{2}, \ldots, p_{k}}$ be a complete $k$-partite graph with vertex set $S_{1} \cup S_{2} \cup \ldots \cup S_{k}, k \geq 2,\left|S_{1}\right|=p_{1},\left|S_{2}\right|=$ $p_{2}, \ldots,\left|S_{k}\right|=p_{k}, p_{1} \geq p_{2} \geq \ldots \geq p_{k} \geq 2$.

The proof of the theorem is distinguished into the following two cases (see Figure 1):

Case 1: $p_{1}>p_{2}$.

There is an edge-coloring of $K_{p_{1}, p_{2}, \ldots, p_{k}}$ using $\sum_{1 \leq i<j \leq k} p_{i} p_{j}-2 \sum_{i=2}^{k} p_{i}+1$ colors such that $K_{p_{1}, p_{2}, \ldots, p_{k}}$
does not contain any rainbow spanning tree $T_{n}$.

Firstly, fix two vertices $v_{1}$ and $v_{2}$ from $S_{1}$ and color all edges incident with $v_{1}$ and $v_{2}$ by some color, say $c_{1}$, that is, $c\left(v_{1} u_{i}\right)=c_{1}$ and $c\left(v_{2} u_{i}\right)=c_{1}$, for all vertices $u_{i} \in V\left(K_{p_{1}, p_{2}, \ldots, p_{k}}\right)-S_{1}$. Since $\left|E\left(K_{p_{1}, p_{2}, \ldots, p_{k}}\right)\right|=$ $\sum_{1 \leq i<j \leq k} p_{i} p_{j}$, the number of remaining edges which are not colored is $\sum_{1 \leq i<j \leq k} p_{i} p_{j}-2 \sum_{i=2}^{k} p_{i}$. Then, color all other edges of $K_{p_{1}, p_{2}, \ldots, p_{k}}$ using $\sum_{1 \leq i<j \leq k} p_{i} p_{j}-2 \sum_{i=2}^{k} p_{i}$ colors such that each appears on one edge. Assume that there is a rainbow spanning tree $T_{n}$ of $K_{p_{1}, p_{2}, \ldots, p_{k}}$ in this coloring, and then the spanning tree $T_{n}$ must contain two edges with the same color $c_{1}$, one incident with $v_{1}$ and the other incident with $v_{2}$, a contradiction. Thus,

$$
A R\left(K_{p_{1}, p_{2}, \ldots, p_{k}}, \mathscr{T}_{n}\right) \geq \sum_{1 \leq i<j \leq k} p_{i} p_{j}-2 \sum_{i=2}^{k} p_{i}+1 .
$$

Case 2: $p_{1}=p_{2}$.

If we use $\sum_{1 \leq i<j \leq k} p_{i} p_{j}-2 \sum_{i=2}^{k} p_{i}+2$ different colors to color the edges of $K_{p_{1}, p_{2}, \ldots, p_{k}}$, then the $K_{p_{1}, p_{2}, \ldots, p_{k}}$ does not contain any rainbow spanning tree $T_{n}$.

Fix vertices $v_{1}$ from $S_{1}$ and $u_{1}$ from $S_{2}$. Firstly, color the edges incident with $v_{1}$ and $u_{1}$ by color $c_{1}$, that is, $c\left(v_{1} w_{1}\right)=c_{1}$, for all vertices $w_{1} \in V\left(K_{p_{1}, p_{2}, \ldots, p_{k}}\right)-S_{1}$ and $c\left(u_{1} w_{2}\right)=c_{1}$, for all vertices $w_{2} \in V\left(K_{p_{1}, p_{2}, \ldots, p_{k}}\right)-S_{2}$, then color the remaining edges of $K_{p_{1}, p_{2}, \ldots, p_{k}}$ using $\sum_{1 \leq i<j \leq k} p_{i} p_{j}-$ $2 \sum_{i=2}^{k} p_{i}+1$ colors such that each appears on one edge, and the number of colors is $\sum_{1 \leq i<j \leq k} p_{i} p_{j}-2 \sum_{i=2}^{k} p_{i}+2$. Now, every spanning tree $T_{n}$ of $K_{p_{1}, p_{2}, \ldots, p_{k}}$ has at least two edges of the same color $c_{1}$. Thus,

$$
A R\left(K_{p_{1}, p_{2}, \ldots, p_{k}}, \mathscr{T}_{n}\right) \geq \sum_{1 \leq i<j \leq k} p_{i} p_{j}-2 \sum_{i=2}^{k} p_{i}+2 .
$$

Theorem 2. If $\left|S_{1}\right|=p_{1},\left|S_{2}\right|=p_{2}, \ldots,\left|S_{k}\right|=p_{k}, \quad p_{1} \geq p_{2} \geq \ldots$ $\geq p_{k} \geq 2, k \geq 2$, then

$$
A R\left(K_{p_{1}, p_{2}, \ldots, p_{k}}, \mathscr{T}_{n}\right) \leq \sum_{1 \leq i<j \leq k} p_{i} p_{j}-\sum_{i=2}^{k} p_{i} .
$$

Proof. We consider an arbitrary edge-coloring of $K_{p_{1}, p_{2}, \ldots, p_{k}}$ using $\sum_{1 \leq i<j \leq k} p_{i} p_{j}-\sum_{i=2}^{k} p_{i}+1$ different colors. We only show that there is a spanning tree $T_{n}$ of $K_{p_{1}, p_{2}, \ldots, p_{k}}$. We choose a representing subgraph $G$ from $K_{p_{1}, p_{2}, \ldots, p_{k}}$ with $|E(G)|=\sum_{1 \leq i<j \leq k} p_{i} p_{j}-\sum_{i=2}^{k} p_{i}+1$. Note that $K_{p_{1}, p_{2}, \ldots, p_{k}}$ is disconnected by deleting at least $\sum_{i=2}^{k} p_{i}$ edges. Thus, $G$ is connected. $G$ contains a rainbow spanning tree $T_{n}$ since every connected graph has a spanning tree.

The family of all Hamilton cycles in $K_{p_{1}, p_{2}, \ldots, p_{k}}$ is denoted by $\mathscr{C} . A R\left(K_{p_{1}, p_{2}, \ldots, p_{k}}, \mathscr{C}\right)$ is the maximum number of colors in an edge-coloring $K_{p_{1}, p_{2}, \ldots, p_{k}}$ not containing any rainbow Hamilton cycle.

In order to prove our main result, we need the following lemma.

Lemma 1 (Dirac's theorem, see [1]). If $G$ is a graph on $n \geq 3$ vertices such that $\delta(G) \geq(n / 2)$, then $G$ is Hamiltonian.

Theorem 3. Let $K_{p_{1}, p_{2}, \ldots, p_{k}}$ be a complete $k$-partite graph with $p_{1} \geq p_{2} \geq \ldots \geq p_{k} \geq 2$ and $k \geq 2 ;$ if $\sum_{i=2}^{k} p_{i} \geq p_{1}$, then $K_{p_{1}, p_{2}, \ldots, p_{k}}$ must have a Hamilton cycle.

Proof. By assumption and the structure of $K_{p_{1}, p_{2}, \ldots, p_{k}}$, it is clear that $\delta\left(K_{p_{1}, p_{2}, \ldots, p_{k}}\right)=\sum_{i=2}^{k} p_{i}$, and according to Dirac's Theorem, $\quad \sum_{i=2}^{k} p_{i} \geq\left(\left|V\left(K_{p_{1}, p_{2}, \ldots, p_{k}}\right)\right| / 2\right)=\left(\sum_{i=1}^{k} p_{i} / 2\right), \quad K_{p_{1}, p_{2}, \ldots, p_{k}}$ have a Hamilton cycle. In fact, $\sum_{i=2}^{k} p_{i} \geq\left(\sum_{i=1}^{k} p_{i} / 2\right)$, namely, $\sum_{i=2}^{k} p_{i} \geq p_{1}$. The proof is finished.

Theorem 4. If $p_{1} \geq p_{2} \geq \ldots \geq p_{k} \geq 2, k \geq 2, \quad \sum_{i=2}^{k} p_{i} \geq p_{1}$, then

$$
A R\left(K_{p_{1}, p_{2}, \ldots, p_{k}}, \mathscr{C}\right) \geq \sum_{1 \leq i<j \leq k} p_{i} p_{j}-\sum_{i=2}^{k} p_{i}+1 .
$$

Proof. By assumption and Theorem $4, K_{p_{1}, p_{2}, \ldots, p_{k}}$ is clear Hamiltonian. Now, we show that there is an edge-coloring of $K_{p_{1}, p_{2}, \ldots, p_{k}}$ using $\sum_{1 \leq i<j \leq k} p_{i} p_{j}-\sum_{i=2}^{k} p_{i}+1$ colors such that $K_{p_{1}, p_{2}, \ldots, p_{k}}$ does not contain rainbow Hamilton cycle. Firstly, fix any one vertex $v$ from $S_{1}$, color all the edges incident with $v$ by color $c_{1}$, and then color all other edges of $K_{p_{1}, p_{2}, \ldots, p_{k}}$ using $\sum_{1 \leq i<j \leq k} p_{i} p_{j}-\sum_{i=2}^{k} p_{i}$ colors such that each appears on one edge. Note that every Hamilton cycle of $K_{p_{1}, p_{2}, \ldots, p_{k}}$ must contain two edges incident with $v$, and the 


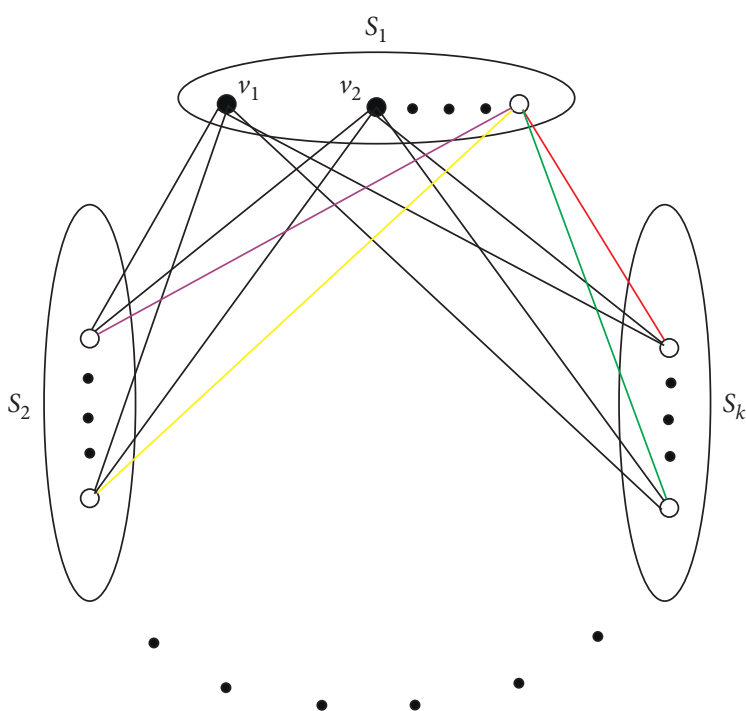

(a)

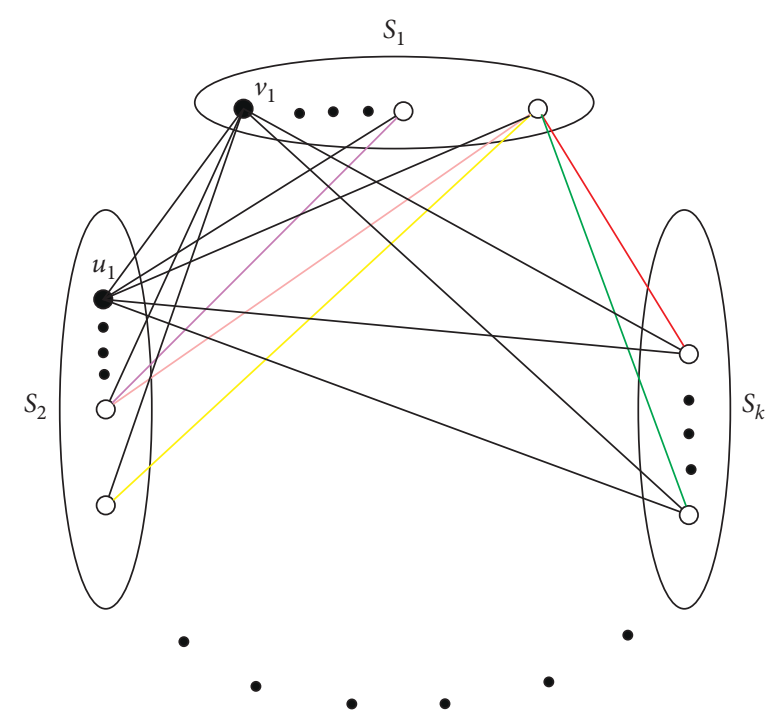

(b)

Figure 1: Two ways of coloring in case 1 and case 2.

two edges have the same color $c_{1}$. So, $K_{p_{1}, p_{2}, \ldots, p_{k}}$ has no rainbow Hamilton cycle. Thus,

$$
A R\left(K_{p_{1}, p_{2}, \ldots, p_{k}}, \mathscr{C}\right) \geq \sum_{1 \leq i<j \leq k} p_{i} p_{j}-\sum_{i=2}^{k} p_{i}+1 .
$$

In order to prove the next main theorem, we need the following definition.

Let $G$ be a $k$-partite graph with vertex set $S_{1} \cup S_{2} \cup \ldots \cup S_{k}$, if there are two vertices $u \in S_{i}$ and $v \in S_{j}$, $i \neq j, \quad i, j \in\{1,2, \ldots, k\}, \quad u v \notin E(G)$, with $d_{G}(u)+d_{G}$ $(v) \geq(k-1) p+1$, then add an edge $u v$ to $E(G)$. The closure of $G$ is the graph obtained from $G$ by repeating this step until there are no such pair of vertices, denoted by $c(G)$.

Lemma 2 (see [1]). G is a simple graph, and then $G$ contains a Hamilton cycle if and only if its closure $c(G)$ is Hamiltonian.

Theorem 5. Let $G$ be a k-partite graph with $\left|S_{1}\right|=\left|S_{2}\right|=\ldots=\left|S_{k}\right|=p \geq 2$. Suppose $d_{1}^{j}, d_{2}^{j}, \ldots, d_{p}^{j}$ are the vertex degrees of $S_{j}$ of $G$, all in nondecreasing order, where $1 \leq j \leq k, k \geq 2$. If $d_{i}^{j}>i$ for each $i \leq((k-1) p / 2)$, then $G$ contains a Hamilton cycle.

Proof. By Lemma 2, we only need to prove that $c(G)$ is Hamiltonian. $c(G)$ is a $k$-partite graph with vertex set $S_{1} \cup S_{2} \cup \ldots \cup S_{k}$. Suppose $d_{1}^{(j)}, d_{2}^{(j)}, \ldots, d_{p}^{(j)}$ are the vertex degrees of $S_{j}$ of $c(G)$, all in nondecreasing order, where $1 \leq j \leq k, k \geq 2, p \geq 2 . d_{i}^{(j)} \geq d_{i}^{j}$ for $1 \leq i \leq p, 1 \leq j \leq k$. To the contrary, suppose $c(G)$ contains no Hamilton cycle, then $c(G)$ is not a complete $k$-partite graph. Let $u$ and $v$ be two vertices in $c(G), u \in S_{i}, \quad v \in S_{j}, \quad i \neq j, \quad u v \notin E(G)$, and $d_{c(G)}(u)+d_{c(G)}(v) \leq(k-1) p$. If $d_{c(G)}(u) \leq d_{c(G)}(v)$, set $i=d_{c(G)}(u) \leq((k-1) p / 2)$. $(k-1) p-d_{c(G)}(v) \geq d_{c(G)}(u)=i, c(G)$ contains at least $(k-1) p-d_{c(G)}(v) \geq i$ vertices that are not adjacent to $v$, and each of which has degree at most $d_{c(G)}(u)=i$. Thus, we can find some vertex $w$ whose degree is at most $d_{c(G)}(u)=i$ in $c(G)$, which implies that $i \geq d_{c(G)}(w) \geq d_{G}(w)$, that is $i \geq d_{i}^{(j)} \geq d_{i}^{j}$, a contradiction.

Theorem 6. If $p_{1} \geq p_{2} \geq \ldots \geq p_{k}=p \geq 2, k \geq 2$, then

$$
A R\left(K_{p_{1}, p_{2}, \ldots, p_{k}}, \mathscr{C}\right)=\left(\frac{k(k-1)}{2}\right) p^{2}-(k-1) p+1 .
$$

Proof. By Theorem 4, we can easily prove the lower bound. We consider an arbitrary edge-coloring of $K_{p_{1}, p_{2}, \ldots, p_{k}}$ using $(k(k-1) / 2) p^{2}-(k-1) p+2$ different colors, and we will find a rainbow Hamilton cycle in $K_{p_{1}, p_{2}, \ldots, p_{k}}$. We choose a representing subgraph $G$ with $|E(G)|=(k(k-1) / 2) p^{2}-$ $(k-1) p+2$. Let $d_{1}^{j}, d_{2}^{j}, \ldots, d_{p}^{j}$ be the vertex degrees of $S_{j}$ of $G$, all in nondecreasing order. If $d_{i}^{j}>i$ for each $i \leq((k-1) p / 2), 1 \leq j \leq k$, then $G$ must contain a Hamilton cycle. If not, we assume that there exists $i^{*}$ such that $d_{i^{*}}^{j} \leq i^{*}$, $i^{*} \leq((k-1) p / 2)$. Without loss of generality, we assume that $d_{1}^{1} \leq d_{1}^{2} \leq \ldots \leq d_{1}^{k}$ and $d_{i^{*}}^{1} \leq i^{*}$. We have $i^{*} \cdot i^{*}+\left(p-i^{*}\right)(k-$ 1) $p+((k-1)(k-2) / 2) p^{2} \geq(k(k-1) / 2) p^{2}-(k-1) p+2=$ $|E(G)|$, that is, $\left(i^{*}\right)^{2}-p(k-1) i^{*}+p(k-1)-2 \geq 0$. Set $\left.f(i)=i^{2}-p(k-1) i+p(k-1)-2=\right](i-(p(k-1)-1))$ $(i-1)-1, \quad f(1)=-1<0, \quad f(2)=2-p(k-1)<0, \quad f((k$ $-1) p / 2) \leq f(p(k-1)-1)=-1<0$. We conclude that $f(i)<0$ for each $1 \leq i \leq((k-1) p / 2)$. Thus, we have $d_{i}^{j}>i$ for each $i \leq((k-1) p / 2), 1 \leq j \leq k$. By Theorem $7, G$ must have a rainbow Hamilton cycle.

A matching in a graph is a set of nonadjacent edges. A perfect matching $M$ is a matching which saturates every vertex of the graph. The family of all perfect matchings 
$K_{p_{1}, p_{2}, \ldots, p_{k}}$ is denoted by $\mathscr{M} . A R\left(K_{p_{1}, p_{2}, \ldots, p_{k}}, \mathscr{M}\right)$ is the maximum number of colors in an edge-coloring of $K_{p_{1}, p_{2}, \ldots, p_{k}}$ not containing any rainbow perfect matching.

In [9], it has been shown that $A R\left(K_{m, n}, k K_{2}\right)=$ $m(k-2)+1, m \geq n \geq k \geq 3$, which is the maximum numbers of colors in an edge-coloring of $K_{m, n}$ that contains no rainbow $k K_{2}$. Now, we consider the maximum numbers of colors in an edge-coloring of $K_{p_{1}, p_{2}, \ldots, p_{k}}$ not containing any rainbow perfect matching.

Tutte gives the sufficient and necessary condition of a graph with perfect matchings.

Lemma 3 (Tutte's theorem, see [1]). A graph $G$ has a perfect matching if and only if $C_{0}(G-S) \leq|S|$, for all $S \subseteq V(G)$, where $C_{0}(G-S)$ is the number of odd components of $G-S$.

According to Tutte's theorem, we give the following sufficient condition that completes $k$-partite graph $K_{p_{1}, p_{2}, \ldots, p_{k}}$ have a perfect matching.

Theorem 7. If $p_{1} \geq p_{2} \geq \ldots \geq p_{k} \geq 1, k \geq 2, \sum_{i=1}^{k} p_{i}$ is even and $\sum_{i=2}^{k} p_{i} \geq p_{1}$, then the complete $k$-partite graph $K_{p_{1}, p_{2}, \ldots, p_{k}}$ must have a perfect matching.

Proof. Let $S$ be a subset of $V\left(K_{p_{1}, p_{2}, \ldots, p_{k}}\right)$, and we consider the following three cases according to the cardinality of $S$.

Case 1: $0 \leq|S|<\sum_{i=2}^{k} p_{i}$.

Note that $K_{p_{1}, p_{2}, \ldots, p_{k}}$ is disconnected by deleting at least $\sum_{i=2}^{k} p_{i}$ vertices. For $0 \leq|S|<\sum_{i=2}^{k} p_{i}$, it is clear that $K_{p_{1}, p_{2}, \ldots, p_{k}}-S$ is connected. If $\left|V\left(K_{p_{1}, p_{2}, \ldots, p_{k}}-S\right)\right|$ is even, then $C_{0}\left(K_{p_{1}, p_{2}, \ldots, p_{k}}-S\right)=0 \leq|S|$, and if $\left|V\left(K_{p_{1}, p_{2}, \ldots, p_{k}}-S\right)\right|$ is odd, then $C_{0}\left(K_{p_{1}, p_{2}, \ldots, p_{k}}-S\right)=1$, by assuming that $\sum_{i=1}^{k} p_{i}$ is even; thus, $|S|$ is odd and $|S| \geq 1$ by the parity. So, $C_{0}\left(K_{p_{1}, p_{2}, \ldots, p_{k}}-S\right) \leq|S|$. By Lemma $3, K_{p_{1}, p_{2}, \ldots, p_{k}}$ has a perfect matching.

Case 2: $|S|=\sum_{i=2}^{k} p_{i}$.

If $|S|=\sum_{i=2}^{k} p_{i}, C_{0}\left(K_{p_{1}, p_{2}, \ldots, p_{k}}-S\right)=p_{1} \leq \sum_{i=2}^{k} p_{i}=|S|$, which meets Lemma 3 , then $K_{p_{1}, p_{2}, \ldots, p_{k}}$ has a perfect matching.

Case 3: $|S|>\sum_{i=2}^{k} p_{i}$.

If $S>\sum_{i=2}^{k} p_{i}, \quad C_{0}\left(K_{p_{1}, p_{2}, \ldots, p_{k}}-S\right)=\sum_{i=1}^{k} p_{i}-|S|<p_{1}$ $<|S|$, which also meets Lemma 9 , then $K_{p_{1}, p_{2}, \ldots, p_{k}}$ has a perfect matching.

Therefore, if $\sum_{i=1}^{k} p_{i}$ is even and $\sum_{i=2}^{k} p_{i} \geq p_{1}, K_{p_{1}, p_{2}, \ldots, p_{k}}$ must have a perfect matching.

In this section, we consider the anti-Ramsey problem of perfect matching in complete $k$-partite graph $K_{p_{1}, p_{2}, \ldots, p_{k}}$.

Theorem 8. If $p_{1} \geq p_{2} \geq \ldots \geq p_{k} \geq 2, k \geq 2, \sum_{i=1}^{k} p_{i}$ is even and $\sum_{i=2}^{k} p_{i} \geq p_{1}$, then

$$
A R\left(K_{p_{1}, p_{2}, \ldots, p_{k}}, \mathscr{M}\right) \geq \sum_{1 \leq i<j \leq k} p_{i} p_{j}-2 \sum_{i=2}^{k} p_{i}+1 .
$$

Proof. The known conditions clearly met that $K_{p_{1}, p_{2}, \ldots, p_{k}}$ must have a perfect matching by Theorem 9. Now, we firstly show that there is an edge-coloring of $K_{p_{1}, p_{2}, \ldots, p_{k}}$ using $\sum_{1 \leq i<j \leq k} p_{i} p_{j}-2 \sum_{i=2}^{k} p_{i}+1$ colors such that $K_{p_{1}, p_{2}, \ldots, p_{k}}$ does not contain any rainbow perfect matching $M$. Fix two vertices $v_{1}$ and $v_{2}$ from $S_{1}$, color the edges incident with $v_{1}$ and $v_{2}$ by coloring $c_{1}$, and color the remaining edges of $K_{p_{1}, p_{2}, \ldots, p_{k}}$ using $\sum_{1 \leq i<j \leq k} p_{i} p_{j}-2 \sum_{i=2}^{k} p_{i}$ colors such that each appears on one edge. It is clear that there is no rainbow perfect matching in $K_{p_{1}, p_{2}, \ldots, p_{k}}$. So, we have

$$
A R\left(K_{p_{1}, p_{2}, \ldots, p_{k}}, \mathscr{M}\right) \geq \sum_{1 \leq i<j \leq k} p_{i} p_{j}-2 \sum_{i=2}^{k} p_{i}+1 .
$$

Theorem 9. If $p_{1} \geq p_{2} \geq \ldots \geq p_{k}=p \geq 2, k \geq 2, k p$ is even, then

$$
A R\left(K_{p_{1}, p_{2}, \ldots, p_{k}}, \mathscr{M}\right) \leq\left(\frac{k(k-1)}{2}\right) p^{2}-(k-1) p+1 .
$$

Proof. We consider an arbitrary edge-coloring of $K_{p_{1}, p_{2}, \ldots, p_{k}}$ using $(k(k-1) / 2) p^{2}-(k-1) p+2$ different colors, and we choose a representing subgraph $G$ from $K_{p_{1}, p_{2}, \ldots, p_{k}}$. By the proof of Theorem 6, we know that $G$ must have a rainbow Hamilton cycle $C$. Then, we can find a rainbow perfect matching from $C$ since the number of vertices in $K_{p_{1}, p_{2}, \ldots, p_{k}}$ is even. So,

$$
A R\left(K_{p_{1}, p_{2}, \ldots, p_{k}}, \mathscr{M}\right)<\left(\frac{k(k-1)}{2}\right) p^{2}-(k-1) p+2 .
$$

The proof is completed.

\section{Data Availability}

The data used to support the findings of this study are available from the corresponding author upon request.

\section{Conflicts of Interest}

The authors declare that there are no conflicts of interest regarding the publication of this paper.

\section{Acknowledgments}

The research and publication of our article was funded by the National Natural Science Foundation of China (61662079). H. Bian was supported by the National Natural Science Foundation of China (11761070). H. Z. Yu was supported by the National Natural Science Foundation of China (61662079). J. L. Ding was supported by the 2020 Postgraduate Innovation Project of Xinjiang.

\section{References}

[1] J. A. Bondy and U. S. R. Murty, Graph Theory, Springer, Berlin, Germany, 2008.

[2] P. ErdÖs, M. Simonovits, and V. T. SÓs, "Anti-Ramsey theorems," Colloquia Mathematica Societatis János Bolyai, vol. 10, no. 1, pp. 633-643, 1973. 
[3] J. J. Montellano-Ballesteros and V. Neumann-Lara, "An antiramsey theorem on cycles," Graphs and Combinatorics, vol. 21, no. 3, pp. 343-354, 2005.

[4] Z. Jin and X. Li, "Anti-Ramsey numbers for graphs with independent cycles," The Electronic Journal of Combinatorics, vol. 16, no. 1, p. 1200, 2009.

[5] T. Jiang, "Edge-Colorings with No large polychromatic stars," Graphs and Combinatorics, vol. 18, no. 2, pp. 303-308, 2002.

[6] A. Bialostocki and W. Voxman, "On the anti-Ramsey numbers for spanning trees," Bulletin of the ICA, vol. 32, pp. 23-26, 2001.

[7] H. Chen, X. Li, and J. Tu, "Complete solution for the rainbow numbers of matchings," Discrete Mathematics, vol. 309, no. 10, pp. $3370-3380,2009$.

[8] R. Haas and M. Young, "The anti-Ramsey number of perfect matching," Discrete Mathematics, vol. 312, no. 5, pp. 933-937, 2012.

[9] X. Li, J. Tu, and Z. Jin, "Bipartite rainbow numbers of matchings," Discrete Mathematics, vol. 309, no. 8, pp. 2575-2578, 2009.

[10] M. Axenovich, T. Jiang, and A. Kündgen, "Bipartite antiRamsey numbers of cycles," Journal of Graph Theory, vol. 47, no. 1, pp. 9-28, 2004.

[11] Z. Jin and L. Li, "Edge-colorings of complete bipartite graphs without large rainbow trees," Ars Combinatoria-Waterloo Then Winnipeg-, vol. 111, pp. 75-84, 2013.

[12] R. Gu, J. Li, and Y. Shi, "Anti-ramsey numbers of paths and cycles in hypergraphs," SIAM Journal on Discrete Mathematics, vol. 34, no. 1, pp. 271-307, 2020.

[13] M. Axenovich, H. Harborth, A. Kemnitz, M. Möller, and I. Schiermeyer, "Rainbows in the hypercube," Graphs and Combinatorics, vol. 23, no. 2, pp. 123-133, 2007.

[14] S. Jendrol, I. Schirmeyer, and J. H. Tu, "Rainbow numbers for matchings in plane triangulations," Discrete Math, vol. 331, pp. 158-164, 2014.

[15] Y. Lan, Y. Shi, and Z.-X. Song, "Planar anti-Ramsey numbers of paths and cycles," Discrete Mathematics, vol. 342, no. 11, pp. 3216-3224, 2019. 